\title{
Detection of Two Carbon-Chain-Rich Cores; CB130-3 and L673-SMM4
}

\author{
Tomoya HIROTA \\ National Astronomical Observatory of Japan, Osawa 2-21-1, Mitaka, Tokyo 181-8588, \\ Japan; \\ tomoya.hirota@nao.ac.jp \\ Takeshi SAKAI \\ Institute of Astronomy, The University of Tokyo, Osawa 2-21-1, Mitaka, Tokyo 181-0015, \\ Japan \\ and \\ Nami SAKAI, Satoshi YAMAMOTO \\ Department of Physics and Research Center for the Early Universe, \\ The University of Tokyo, Hongo 7-3-1, Bunkyo-ku, Tokyo 113-0033, Japan
}

\begin{abstract}
We have found two dense cores, CB130-3 and L673-SMM4, where the carbonchain molecules are extremely abundant relative to $\mathrm{NH}_{3}$, during a survey observation of radio emission lines of $\mathrm{CCS}, \mathrm{HC}_{3} \mathrm{~N}, \mathrm{HC}_{5} \mathrm{~N}$, and $\mathrm{NH}_{3}$ toward dark cloud cores. Judging from the low $\mathrm{NH}_{3} / \mathrm{CCS}$ ratios, they are possible candidates for "Carbon-Chain-Producing Regions (CCPRs)" recognized as chemically young dark cloud cores. The deuterium fractionation ratios $\mathrm{DNC} / \mathrm{HN}^{13} \mathrm{C}$ in $\mathrm{CB} 130-3$ and L673-SMM4 are found to be $1.28_{-0.05}^{+0.27}$ and $1.96_{-0.01}^{+0.32}$, respectively, which are comparable to or slightly higher than those in CCPRs found previously. We suggest that the dense cores of CB130-3 and L673-SMM4 are analogous to CCPRs but their chemical evolutionary phase would be slightly older than those of the dense cores in the Taurus region.
\end{abstract}

Subject headings: ISM:abundances — ISM:Individual objects (CB130-3, L673SMM4) — ISM:Molecules — radio lines: ISM 


\section{Introduction}

Since the discovery of carbon-chain molecules, their spectral lines have been employed as one of the best tracers of dense molecular cloud cores (e.g., Suzuki et al. 1992). Both abundances and distributions of carbon-chain molecules are found to be anti-correlated with those of $\mathrm{NH}_{3}$ and $\mathrm{N}_{2} \mathrm{H}^{+}$. The representative sources observed extensively are TMC-1 (Hirahara et al. 1992) and L1544 (Ohashi et al. 1999) in the Taurus Molecular Cloud. The anti-correlation can be interpreted as a consequence of chemical evolution of dark cloud cores, because carbon-chain molecules and nitrogen-bearing inorganic molecules are expected to be abundant in the early and late stages of chemical evolution, respectively (Suzuki et al. 1992; Bergin \& Langer 1997; Aikawa et al. 2001, 2003, 2005). Thus, dense cores where carbonchain molecules are abundant are thought to be in a very early phase of chemical and dynamical evolution.

According to the pioneering survey of the CCS lines by Suzuki et al. (1992), four dense cores were identified where extraordinarily intense spectra of carbon-chain molecules were detected in spite of very weak $\mathrm{NH}_{3}$ lines. These four sources, L1495B, L1521B, L1521E, and the cyanopolyyne peak of TMC-1, are named as "Carbon-Chain-Producing Regions" (hereafter called CCPR). Until our recent discovery of another candidate L492 in the Aquila rift region (Hirota \& Yamamoto 2006; Hirota et al. 2009), CCPRs were identified only in the Taurus Molecular Cloud (Hirota et al. 2002, 2004; Tafalla \& Santiago 2004). Based on the previous survey observations, the fraction of CCPRs among all dense cores is found to be about 10\% (Suzuki et al. 1992; Hirota et al. 2009). This means that the timescale staying in the CCPR phase is expected to be shorter than a typical lifetime of dense cores by a factor of 10. This is consistent with the idea that the evolutionary stage of CCPRs is relatively younger than other typical dark cloud cores. Thus, CCPRs would be the best targets to investigate early stages for the gas-phase chemistry under extremely low degree of depletion, as well as dynamical state of dense cores just after dense core formation.

However, CCPRs have rarely been detected outside the Taurus region. According to Hirota et al. (2009), there could be possible chemical differentiation among molecular clouds including the Taurus, Ophiuchus, and Aquila regions. For instance, detection rates of carbonchain molecules are found to be systematically higher in the Taurus region than those in Ophiuchus. This implies that the average duration time of the starless core phase is different from cloud to cloud. However, we cannot rule out the possibility that the higher detection rate of CCPRs is due to some specific nature in the Taurus region. In order to examine this possibility, a search for further CCPR candidates other than in the Taurus region is useful.

With this motivation, we carried out a survey observation of the $\mathrm{CCS}, \mathrm{HC}_{3} \mathrm{~N}, \mathrm{HC}_{5} \mathrm{~N}$,

and $\mathrm{NH}_{3}$ lines toward more than 100 dark cloud cores (Hirota et al. 2009, 2011). As a 
result, we detected two dense cores, CB130-3 and L673-SMM4, which show remarkably intense spectra of carbon-chain molecules. CB130-3 is identified as an optical dark cloud core by Lee \& Myers (1999) in the Aquila rift region at the distance of 200 pc. CB130-3 resides in the globular filament GF4 (Schneider \& Elmegreen 1979), where another CCPR, L492, is also located as shown in Figure 1. L673-SMM4 is one of the dust continuum peaks (Visser et al. 2002) in the dark cloud L673 located in the Cloud B region at the distance of $300 \mathrm{pc}$ (Dame et al. 1987). In this paper, we present results of our intensive observations of newly found CCPR candidates. Detail for the survey observation will be described in the forthcoming paper (Hirota et al. 2011).

\section{Observations}

We first conducted the survey observation with the $45 \mathrm{~m}$ radio telescope at Nobeyama Radio Observatory (NRO) in 2008 May and 2009 April. The CCS $\left(J_{N}=4_{3}-3_{2}\right), \mathrm{HC}_{3} \mathrm{~N}(J=5$ $4)$, and $\mathrm{HC}_{5} \mathrm{~N}(J=17-16)$ lines in the $45 \mathrm{GHz}$ region were observed simultaneously with a Superconductor-Insulator-Superconductor (SIS) mixer receiver, whose system temperature was $190-260 \mathrm{~K}$. The main-beam efficiency $\left(\eta_{m b}\right)$ and the beam size (HPBW) were 0.7 and $37^{\prime \prime}$, respectively. During the survey observation, we serendipitously found strong emissions of the CCS, $\mathrm{HC}_{3} \mathrm{~N}$, and $\mathrm{HC}_{5} \mathrm{~N}$ lines toward CB130-3 and L673-SMM4. Subsequently, we made mapping observations of the lines in these two sources with a grid spacing of $40^{\prime \prime}$. The source coordinates are listed in Table 1. In addition, we observed the molecular lines in the 72-93 GHz band as listed in Table 2 toward the CCS peak in each core. One of the SIS mixer receivers equipped with the NRO $45 \mathrm{~m}$ telescope was used for the observations of the $72-93 \mathrm{GHz}$ lines. The system temperatures ranged from 180 to $400 \mathrm{~K}$, depending on the frequencies. The main-beam efficiency and the beam size were approximately 0.44 and $20^{\prime \prime}$, respectively, although these values depend on the observed frequencies. For all of the frequency bands, acousto-optical radio spectrometers with the frequency resolution of $37 \mathrm{kHz}$ were used as the back end. Observations were performed in the position-switching mode with the off position of $10^{\prime}$ toward the azimuth direction. The antenna temperatures were calibrated by using the chopper-wheel method (Ulich \& Haas 1976). Pointing was checked every two hours by observing the nearby $\mathrm{SiO}$ maser sources, V446 Oph and RR Aql, for observations of CB130-3 and L673-SMM4, respectively, and the pointing accuracy was kept to be better than $5^{\prime \prime} \mathrm{rms}$. The observed data were analyzed with the software package NEWSTAR developed by NRO.

The $\mathrm{NH}_{3}$ lines in the $23 \mathrm{GHz}$ band were also observed with the $100 \mathrm{~m}$ radio telescope

of Max Planck Institute for Radio Astronomy (MPIfR) in Effelsberg. The observations were 
carried out in 2010 February. A dual polarization high electron mobility transistor (HEMT) receiver was employed with the system temperature of 50-90 K. The mapping observations were made with the grid spacing of $40^{\prime \prime}$ in the frequency switching mode. The fast Fourier transform (FFT) spectrometer was used for the back end, of which spectral resolution was set to be $10 \mathrm{kHz}$. The pointing and focusing were checked by observing a continuum emission from the quasar 1741-038. The flux calibration was conducted by observing NGC 7027, whose flux density is calculated to be 5.64 Jy with a program provided by the observatory (e.g., Ott et al. 1994). The data were analyzed with the CLASS software package.

\section{Results}

\subsection{Molecular Abundances}

Observed spectra are shown in Figures 2 and 3 . The intensities of the $\mathrm{CCS}, \mathrm{HC}_{3} \mathrm{~N}$, and $\mathrm{HC}_{5} \mathrm{~N}$ lines toward CB130-3 and L673-SMM4 are found to be remarkably strong, and are almost comparable to those toward the previously known CCPRs (Hirota et al. 2002, 2004; Hirota \& Yamamoto 2006). The peak brightness temperature, line width, and LSR velocity are derived by fitting the Gaussian profile to each spectrum. The results are summarized in Table 2. The line widths and LSR velocities for $\mathrm{HC}_{3} \mathrm{~N}, \mathrm{DNC}$, and $\mathrm{HN}^{13} \mathrm{C}$ are slightly different from one another due to their unresolved hyperfine structures.

Using these line parameters, column densities of $\mathrm{CCS}, \mathrm{HC}_{3} \mathrm{~N}$, and $\mathrm{HC}_{5} \mathrm{~N}$ were calculated by the method which is consistent with that by Suzuki et al. (1992) and the previous paper (Hirota et al. 2009). The dipole moments of $\mathrm{CCS}, \mathrm{HC}_{3} \mathrm{~N}$, and $\mathrm{HC}_{5} \mathrm{~N}$ were assumed to be 2.81, 3.72, and 4.33 D, respectively (Murakami 1990; Lafferty \& Lovas 1978; Alexander et al. 1976). The LTE condition was assumed, and the excitation temperature was fixed to $6.0 \mathrm{~K}$ for CCS, as in the case of other CCPRs (Suzuki et al. 1992; Hirota et al. 2001). Note that the column density of CCS could not be calculated by assuming the excitation temperature of 5.0 K due to the high brightness temperature for CB130-3. For the $\mathrm{HC}_{3} \mathrm{~N}$ lines in CB1303 and L673-SMM4, we could not detect the satellite hyperfine components $(\Delta F=0)$ to determine the optical depths and the excitation temperatures. Thus, we simply adopted the excitation temperature of $6.5 \mathrm{~K}$ for CB130-3 as employed in the previous papers for

consistency (e.g., Suzuki et al. 1992; Hirota et al. 2009). On the other hand, the higher excitation temperature of $7.5 \mathrm{~K}$ was required to calculate the column density of $\mathrm{HC}_{3} \mathrm{~N}$ in L673-SMM4 due to its exceptionally high brightness temperature. Under these assumptions, the upper limit to the optical depth of the $\mathrm{HC}_{3} \mathrm{~N}$ lines is estimated to be $<3.7$ and $<2.7$ for CB130-3 and L673-SMM4, respectively $(1 \sigma)$. The excitation temperature of $\mathrm{HC}_{5} \mathrm{~N}$ is assumed to be 6.5 K as in the previous studies (Suzuki et al. 1992; Hirota et al. 2009). 
For the $\mathrm{NH}_{3}(1,1)$ line, we derived the excitation temperature and total optical depth based on the hyperfine fitting program in the CLASS software package. As a result, we could determine both parameters toward L673-SMM4, as summarized in Table 3. In contrast, the hyperfine fitting yields the excitation temperature of the $\mathrm{NH}_{3}$ line toward $\mathrm{CB} 130-3$ to be $4.0 \pm 1.2 \mathrm{~K}$, which seems too low in comparison with those in the typical $\mathrm{NH}_{3}$ cores (Suzuki et al. 1992) and other sources in the present study (Table 3). Because of the low signal to noise ratio for the hyperfine components of the $\mathrm{NH}_{3}$ lines toward CB130-3, the fitting result would not be reliable. Thus, we fitted the single Gaussian to the brightest hyperfine component of the $\mathrm{NH}_{3}$ spectrum, and simply adopted the excitation temperature of $6.5 \mathrm{~K}$ for CB130-3 as in the previous papers for consistency (Hirota et al. 2002, 2004; Hirota \& Yamamoto 2006). The line width for CB130-3 derived from the single Gaussian fitting is apparently broadened due to unresolved hyperfine components. We calculated the column density of $\mathrm{NH}_{3}$ under the assumption of the rotation temperature of $10 \mathrm{~K}$ and the ortho-to-para ratio of 1 (the statistical equilibrium value). The dipole moment employed is 1.46 D (Cohen \& Poynter 1974).

The results of the column density calculations are summarized in Table 4, We estimated the uncertainties in the derived column densities as follows. For CCS, $\mathrm{HC}_{3} \mathrm{~N}$, and $\mathrm{HC}_{5} \mathrm{~N}$, the derived column densities differ within $30 \%$ except for $\mathrm{HC}_{3} \mathrm{~N}$ in $\mathrm{L} 673-\mathrm{SMM} 4$, when we change the excitation temperature of $0.5 \mathrm{~K}$, as listed in Table 4. In the case of $\mathrm{HC}_{3} \mathrm{~N}$ in L673-SMM4, the column density differs by a factor of 1.8, because of the higher optical depth. For $\mathrm{NH}_{3}$, we estimated the uncertainties in their column densities by considering the errors of the excitation temperatures in the hyperfine fitting, as summarized in Table 3 . For CB130-3, we changed the excitation temperature of $2 \mathrm{~K}$ to evaluate the possible range of the column density. As a result, the $\mathrm{NH}_{3} / \mathrm{CCS}$ ratio, which is employed as a good indicator of chemical evolutionary phase, would contain uncertainties of about $50 \%$, as summarized in Table 5. Because the assumed excitation temperatures are critical to estimate accurate abundances, further refinement should be made based on multi-transition observations.

The total optical depths for the $\mathrm{N}_{2} \mathrm{H}^{+}(J=1-0)$ lines are derived by the hyperfine fitting to be $2.3 \pm 0.3$ and $6.6 \pm 1.1$ for CB130-3 and L673-SMM4, respectively. The LSR velocities and line widths are also determined, as shown in Table 2. Using these results, we obtained the column densities of $\mathrm{N}_{2} \mathrm{H}^{+}$by assuming the LTE condition as summarized in Table 4 . When we attempted to determine both the excitation temperature and optical depth simultaneously, the least-squares fitting program did not converge with an appropriate solution. Thus, we fixed the excitation temperature of $\mathrm{N}_{2} \mathrm{H}^{+}$to be $5.0 \mathrm{~K}$ to fit all of the hyperfine components (Benson et al. 1998). The uncertainty in the column density is estimated by changing the assumed excitation temperature by $0.5 \mathrm{~K}$. Note that the brightness temperature of the main hyperfine component in L673-SMM4 is underestimated in comparison with 
the observed spectrum (see Table 2 and Figure 2). This indicates higher excitation temperature and lower optical depth in L673-SMM4. If we employed the excitation temperature of $10 \mathrm{~K}$, the fitting result was improved. In this case, the total optical depth was derived to be 1.40 , and the derived column density decreased by a factor of $1.5,8.0 \times 10^{12} \mathrm{~cm}^{-2}$.

For DNC, $\mathrm{HN}^{13} \mathrm{C}$, and $\mathrm{H}^{13} \mathrm{CO}^{+}$, we derived the column densities using the large velocity gradient (LVG) model (Goldreich \& Kwan 1974), as adopted in Hirota et al. (2001, 2003). Details of the analysis are described elsewhere (Hirota \& Yamamoto 2006). Because we observed only the $J=1-0$ lines, the $\mathrm{H}_{2}$ density and the kinetic temperature were fixed to be $1 \times 10^{5} \mathrm{~cm}^{-3}$ and $10 \mathrm{~K}$, respectively. To evaluate the uncertainty in the derived column density, we made the LVG analyses by varying the $\mathrm{H}_{2}$ density from $5 \times 10^{4}$ to $5 \times 10^{5} \mathrm{~cm}^{-3}$. As a result, we confirmed that the $\mathrm{DNC} / \mathrm{HN}^{13} \mathrm{C}$ ratios change only by a factor of 1.2 within the above $\mathrm{H}_{2}$ density range, as shown in Table 5 ,

Table 5 lists the $\mathrm{DNC} / \mathrm{HN}^{13} \mathrm{C}$ and $\mathrm{NH}_{3} / \mathrm{CCS}$ ratios of a few representative sources in addition to those of CB130-3 and L673-SMM4. These two ratios are known to be good indicators of chemical evolutionary stages (Hirota \& Yamamoto 2006). We show a plot of these ratios in Figure 4, as prepared by Hirota et al. (2001). Both the $\mathrm{NH}_{3} / \mathrm{CCS}$ and deuterium fractionation ratios in CB130-3 and L673-SMM4 are systematically lower than those in typical dark cloud cores. In our previous paper (Hirota et al. 2009), we classified CCPRs as dense cores with the $\mathrm{NH}_{3} / \mathrm{CCS}$ ratios lower than 10. The $\mathrm{NH}_{3} / \mathrm{CCS}$ ratios in CB130-3 and L673-SMM4 are slightly higher than the above threshold, indicating that these two sources are close to the evolved prestellar cores such as L1544 (see Table 5). The $\mathrm{NH}_{3} /$ CCS ratio in L492, which is reevaluated in this paper on the basis of the follow-up observation of $\mathrm{NH}_{3}$ (Appendix), is almost comparable to those of CB130-3 and L673-SMM4. Nevertheless, it should be emphasized that the $\mathrm{NH}_{3} / \mathrm{CCS}$ ratios in these cores are still lower than those in typical dark cloud cores.

Note that we here employed the $\mathrm{NH}_{3}$ data obtained with the MPIfR $100 \mathrm{~m}$ telescope, which has higher spatial resolution than the NRO $45 \mathrm{~m}$ telescope, and hence, beam dilution effects would be less significant. In other words, the $\mathrm{NH}_{3} / \mathrm{CCS}$ ratios obtained with the $45 \mathrm{~m}$ telescope are possibly underestimated in comparison with those with the $100 \mathrm{~m}$ telescope. In fact, we confirmed that the brightness temperatures of the $\mathrm{NH}_{3}$ line toward the reference positions $\left(0^{\prime \prime}, 0^{\prime \prime}\right)$ of CB130-3 and L673-SMM4 observed with the NRO $45 \mathrm{~m}$ telescope were $\sim 3$ times weaker than those with the MPIfR $100 \mathrm{~m}$ telescope. From this result, the equivalent FWHM source size of the $\mathrm{NH}_{3}$ cores is estimated to be $\sim 30^{\prime \prime}$, which is consistent with the $\mathrm{NH}_{3}$ core size derived in the mapping observation of this study (Sections 3.2 and 3.3; Figures 5 and 6).

On the basis of the relatively lower $\mathrm{NH}_{3} / \mathrm{CCS}$ and $\mathrm{DNC} / \mathrm{HN}^{13} \mathrm{C}$ ratios in $\mathrm{CB} 130-3$ and 
L673-SMM4, we conclude that CB130-3 and L673-SMM4 would be chemically younger than typical dense cores. This means that they are new candidates for CCPRs outside the Taurus region. On the other hand, these ratios in CB130-3 and L673-SMM4 along with those in L492 are slightly higher than those in CCPRs in the Taurus region, as summarized in Table 5 and Figure 4. Thus, it is likely that CB130-3, L492, and L673-SMM4 are slightly more evolved than other CCPRs in the Taurus region.

\subsection{Molecular Distributions in CB130-3}

We obtained the integrated intensity maps of the $\mathrm{CCS}, \mathrm{HC}_{3} \mathrm{~N}$, and $\mathrm{NH}_{3}$ lines toward CB130-3 as shown in Figure 5, while the $\mathrm{HC}_{5} \mathrm{~N}$ lines are too weak to prepare the map. Both the $\mathrm{HC}_{3} \mathrm{~N}$ and $\mathrm{CCS}$ distributions show a centrally condensed structure without any signature of a dip or a hole caused by molecular depletion, although the distribution of $\mathrm{HC}_{3} \mathrm{~N}$ is slightly more extended along the east-west direction than the CCS distribution.

In contrast, the $\mathrm{NH}_{3}$ distribution shows a double-peaked structure elongating along the east-west direction. The size of the substructure seen in the $\mathrm{NH}_{3}$ map is smaller than the $\mathrm{CCS}$ and $\mathrm{HC}_{3} \mathrm{~N}$ distributions. Since the beam size of the CCS and $\mathrm{HC}_{3} \mathrm{~N}$ observations, $40^{\prime \prime}$, is almost comparable to that of the $\mathrm{NH}_{3}$ observation, this is not due to the spatial resolution. Considering that the observed lines have similar critical densities, this seems to reflect the chemical differentiation.

Such anti-correlation between the distributions of $\mathrm{NH}_{3}$ and carbon-chain molecules like $\mathrm{CCS}$ and $\mathrm{HC}_{3} \mathrm{~N}$ is often found in dense cores (Hirahara et al. 1992; Ohashi et al. 1999). In the starless core L1544, $\mathrm{NH}_{3}$ shows a centrally condensed distribution, whereas CCS has a doughnut-like distribution around it (Ohashi et al. 1999; Tafalla et al. 2002). This means that the central part is more chemically evolved than the outer part. However, the CB130-3 case shows an opposite trend; carbon-chain molecules are centrally condensed while $\mathrm{NH}_{3}$ is distributed outside the carbon-chain molecules. One possibility is that two compact clumps are being formed in the dense core of CB130-3. Chemically evolved clumps traced by the $\mathrm{NH}_{3}$ lines would just be formed by the fragmentation of the parent core traced by the CCS and

$\mathrm{HC}_{3} \mathrm{~N}$ lines. However, we could find no specific velocity/spatial structures suggesting such small scale clumps in the CCS map. Because of the lack of high-resolution dust continuum observations, the density profile and dynamical properties of this core are still unclear. It would be crucial to observe the millimeter and submillimeter dust continuum emissions in order to understand the different distributions of $\mathrm{NH}_{3}$ and CCS in CB130-3.

The line width of the CCPRs is very interesting in relation to their dynamical state. If 
the cores are self-gravitating in the CCPRs, the large line width may be expected. Turbulent motions in the surrounding medium could still be retained in the young core, or the accretion motion of the chemically young material onto dense cores may contribute to the line width. However, we found that the line widths of CB130-3 are not significantly different. The observed line widths for CB130-3 are also similar to those for other CCPRs (Hirota et al. 2002, 2004; Hirota \& Yamamoto 2006). An asymmetric line profile indicating infalling motion has not been detected toward CB130-3 (Lee et al. 1999). It is then suggested that CB130-3 is in dynamically less evolved phase than in other prestellar cores such as L1544, L1498 and another CCPR L492 (Hirota \& Yamamoto 2006). We need more intensive observations to detect the dynamical signatures expected for young clouds.

\subsection{Molecular Distributions in L673-SMM4}

For L673-SMM4, the $\mathrm{CCS}, \mathrm{HC}_{3} \mathrm{~N}, \mathrm{NH}_{3}$, and even $\mathrm{HC}_{5} \mathrm{~N}$ lines are sufficiently intense to map the whole region of the core. The obtained maps are shown in Figure 6. The molecular distributions show an elongated ridge-like structure along the north-south direction. The $\mathrm{HC}_{5} \mathrm{~N}$ line only traces the southern part of the ridge. The peak position for $\mathrm{CCS}, \mathrm{HC}_{3} \mathrm{~N}$, and $\mathrm{HC}_{5} \mathrm{~N}$ is significantly shifted from the dust continuum peak SMM4 (Visser et al. 2002) toward northeast by $\left(40^{\prime \prime}, 40^{\prime \prime}\right)$. One can see the sub-peak at the $\left(80^{\prime \prime}, 160^{\prime \prime}\right)$ position offset from the dust continuum peak in the CCS map. This sub-peak is also evident both in the $\mathrm{HC}_{3} \mathrm{~N}$ and $\mathrm{HC}_{5} \mathrm{~N}$ maps, in which an elongated structure and a weak emission peak, respectively, are detected. In addition, there is an indication of extended structure over the northern edge of the $\mathrm{CCS}$ and $\mathrm{HC}_{3} \mathrm{~N}$ maps.

On the other hand, the $\mathrm{NH}_{3}$ peak is in good agreement with the dust continuum peak, as shown in Figure 7. In addition, we can marginally see the second peak which coincides with the CCS peak position. At the second $\mathrm{NH}_{3}$ peak (i.e., the CCS peak), there is a signature of a sub-peak in the dust continuum map. The dust continuum emission also seems to be around the northern CCS peak. Therefore, the $\mathrm{NH}_{3}$ map well traces the density structure, while the CCS emission shows a possible evidence of depletion at the density peak, or preferentially traces a lower density region.

Although the $\mathrm{NH}_{3}$ emission extends northeastward beyond the $\mathrm{CCS}, \mathrm{HC}_{3} \mathrm{~N}$, and $\mathrm{HC}_{5} \mathrm{~N}$ peaks, its emitting region is smaller than those of carbon-chain molecules. Thus, the $\mathrm{NH}_{3} / \mathrm{CCS}$ ratio shows systematic gradient along the ridge; the $\mathrm{NH}_{3} / \mathrm{CCS}$ ratio is higher in the southern part around the dust continuum peak and decreases toward the northern part. As demonstrated in the TMC-1 ridge (Hirahara et al. 1992), the chemical abundance gradient is explained in terms of a sequential evolution of the filamentary cloud. If this is 
the case, the molecular ridge extending north from the L673-SMM4 would contract first at the southern part of the cloud, and then the contraction would be propagating toward the northern part. We note that the observed line profiles toward the CCS peak in L673-SMM4 are systematically broader than those in CB130-3 and typical dense cores. This might reflect possible effects of star formation such as infalling and/or outflow motions, although the infalling line asymmetry has not been searched for L673-SMM4. Previously, L673-SMM4 is reported to be a starless core (Visser et al. 2002), while a faint protostar candidate has been identified toward the submillimeter dust continuum peak SMM4 based on the mid-infrared observations with the Spitzer Space Telescope (Tsitali et al. 2010). The broader line widths in L673-SMM4 could be affected by the newly formed protostar in the vicinity of the submillimeter peak position. If so, L673-SMM4 is regarded as a chemically young core with a signature of star forming activities.

\section{Discussions}

We have carried out molecular line observations of newly found dark cloud cores, CB1303 and L673-SMM4, showing remarkably intense spectra of the carbon-chain molecules such as $\mathrm{CCS}, \mathrm{HC}_{3} \mathrm{~N}$, and $\mathrm{HC}_{5} \mathrm{~N}$. We have also observed the $\mathrm{NH}_{3}$ lines and other fundamental molecular lines toward these two cores. Judging from the $\mathrm{NH}_{3} / \mathrm{CCS}$ ratios and $\mathrm{DNC} / \mathrm{HN}^{13} \mathrm{C}$ ratios, CB130-3 and L673-SMM4 are analogous to the chemically young dark cloud cores called CCPRs named by Suzuki et al. (1992) but are slightly evolved than those found in the Taurus region.

It should be noted that one of the newly found CCPR candidates, CB130-3, is located close to other dense cores rich in carbon-chain molecules, L492 (Hirota \& Yamamoto 2006; Hirota et al. 2009) and L483 (Hirota et al. 2010). All of these cores belong to an optical dark cloud GF4 (Schneider \& Elmegreen 1979) as shown in Figure 1, We speculate that chemical properties in GF4 in the Aquila rift region are similar to those in the Taurus region.

According to the chemical model calculations (Suzuki et al. 1992), carbon-chain molecules are expected to be abundant in lower extinction and less dense regions where the atomic carbon has not been locked into CO. Thus, it is likely that the CCPRs could be lower extinction and less dense molecular gas clumps formed in the regions in between translucent and dense molecular clouds. In fact, some of the CCPRs are identified as relatively isolated small Bok globules (e.g., L492 and CB130-3 in the Aquila region) unlike the dense molecular cloud such as the Ophiuchus region. However, the CCPRs found in the Taurus and Aquila regions have almost similar $\mathrm{H}_{2}$ densities to other typical dark cloud cores according to the statistical equilibrium calculations for some molecular lines (Hirota et al. 2002, 2004; 
Hirota \& Yamamoto 2006; Aikawa et al. 2005). Although we cannot directly compare the $\mathrm{H}_{2}$ densities in CB130-3 and L673-SMM4, intense spectra of high-density tracers such as $\mathrm{H}^{13} \mathrm{CO}^{+}$imply the $\mathrm{H}_{2}$ densities of an order of $>10^{5} \mathrm{~cm}^{-1}$. Therefore, the CCPRs themselves are not just lower extinction and less dense gas clumps but rather chemically younger dense cores compared with other prestellar cores such as L1544 and L1498. Further observations of these regions including less-dense peripheries around dense cores will be necessary to explore a role of a surrounding environment in formation of dense cores including CCPRs.

Existence of CCPRs outside the Taurus region indicates that the origin of CCPRs is not ascribed to the regional specialty of the Taurus cloud. Hence, our results may strengthen the argument that the evolutionary timescale of dense cores is different from cloud to cloud, as proposed by Hirota et al. (2009) and Sakai et al. (2009). We recently found another type of carbon-chain rich core in the Lupus Molecular Cloud named Lupus-1A (Sakai et al. 2010; Shiino et al. 2011). This source shows extraordinarily intense spectra of $\mathrm{C}_{4} \mathrm{H}$ and longer carbon-chain molecules, while that of CCS is not as bright as in the known CCPRs including CB130-3 and L673-SMM4. All of these sources will be good targets to reveal chemical differentiation among the large-scale molecular cloud complexes. In addition, they can be useful and unique sources to investigate formation and evolution of carbon-chain molecules in dark cloud cores in the chemically young evolutionary phase. They will shed light on the initial state of chemical and dynamical evolution of dark cloud cores by the high-resolution observations with Atacama Large Millimeter/Submillimeter Array (ALMA).

The $45 \mathrm{~m}$ radio telescope is operated by Nobeyama Radio Observatory, a branch of National Astronomical Observatory of Japan (NAOJ). We are grateful to all the staff of Nobeyama Radio Observatory of NAOJ and Effelsberg Observatory of MPIfR for their assistance in observations. TH and NS thank to the Inoue Foundation for Science for the financial support (Research Aid of Inoue Foundation for Science). This study is partly supported by Grant-in-Aid from The Ministry of Education, Culture, Sports, Science and Technology of Japan (No. 21224002 and 21740132).

Facilities: No:45m,Effelsberg.

\section{REFERENCES}

Aikawa, Y., Herbst, E., Roberts, H., \& Caselli, P. 2005, ApJ, 620, 330

Aikawa, Y., Ohashi, N., \& Herbst, E. 2003, ApJ, 593, 906

Aikawa, Y., Ohashi, N., Inutsuka, S., Herbst, E., \& Takakuwa, S. 2001, ApJ, 552, 639 
Alexander, A. J., Kroto, H. W., \& Walton, D. R. M. 1976, J. Mol. Spectrosc., 62, 175

Benson, P. J., Caselli, P., \& Myers, P. C. 1998, ApJ, 506, 743

Bergin, E. A. \& Langer, W. D. 1997, ApJ, 486, 316

Blackman, G. L., Brown, R. D., Godfrey, P. D., \& Gunn, H. I. 1976, Nature, 261, 395

Cohen, E. A., \& Poynter, R. L. 1974, J. Mol. Spectrosc., 53, 131

Dame, T. M. et al. 1987, ApJ, 322, 706

Di Francesco, J., Johnstone, D., Kirk, H., MacKenzie, T., \& Ledwosinska, E. 2008, ApJS, 175,277

Dobashi, K. 2011, PASJ, 63, S1

Goldreich, P., \& Kwan, J. 1974, ApJ, 189, 441

Haese, N. N., \& Woods, R. C. 1979, Chem. Phys. Lett., 61, 396

Havenith, M., Zwart, E., Meerts, W. L., ter Meulen, J. J. 1990, J. Chem. Phys., 93, 8446

Hirahara, Y. et al. 1992, ApJ, 394, 539

Hirota, T., Ikeda, M., \& Yamamoto, S. 2001, ApJ, 547, 814

Hirota, T., Ikeda, M., \& Yamamoto, S. 2003, ApJ, 594, 859

Hirota, T., Ito, T., \& Yamamoto, S. 2002, ApJ, 565, 359

Hirota, T., Maezawa, H., \& Yamamoto, S. 2004, ApJ, 617, 399

Hirota, T., \& Yamamoto, S. 2006, ApJ, 646, 258

Hirota, T., Ohishi, M., \& Yamamoto, S. 2009, ApJ, 699, 585

Hirota, T., Sakai, N., \& Yamamoto, S. 2010, ApJ, 720, 1370

Hirota, T. et al. 2011, in preparation

Lafferty, W. J. \& Lovas, F. J. 1978, J. Phys. Chem. Ref. Data, 7, 441

Lee, C. W., \& Myers, P. C. 1999, ApJS, 123, 233

Lee, C. W., Myers, P. C., \& Tafalla, M. 1999, ApJ, 526, 788 
Murakami, A. 1990, ApJ, 357, 288

Ohashi, N., Lee, S. W., Wilner, D. J., \& Hayashi, M. 1999, ApJL, 518, L41

Ott, M., Witzel, A., Quirrenbach, A., Krichbaum, T. P., Standke, K. J., Schalinski, C. J., \& Hummel, C. A. 1994, A\&A, 284, 331

Sakai, N., Sakai, T., Hirota, T., Burton, M. G., \& Yamamoto, S. 2009, ApJ, 697, 769

Sakai, N., Shiino, T., Hirota, T., Sakai, T., \& Yamamoto, S. 2010, ApJ, 718, L49

Schneider, S. \& Elmegreen, B. G. 1979, ApJS, 41, 87

Shiino, T. et al. 2011, in preparation

Suzuki, H., Yamamoto, S., Ohishi, M., Kaifu, N., Ishikawa, S., Hirahara, Y., \& Takano, S. 1992, ApJ, 392, 551

Tafalla, M., Myers, P. C., Caselli, P., Walmsley, C. M., \& Comito, C. 2002, ApJ, 569, 815

Tafalla, M., \& Santiago, J. 2004, A\&A, 414, L53

Tsitali, A. E., Bourke, B. L., Peterson, D. E., Myers, P. C., Dunham, M. M., Evans, N. J. II., Huard, T. L. 2010, ApJ, 725, 2461

Ulich, B. L. \& Haas, R. W. 1976, ApJS, 30, 247

Visser, A. E., Richer, J. S., \& Chandler, C. J. 2002, AJ, 124, 2756

Yamamoto, S., Shuji, S., Kawaguchi, S., Chikada, Y., Suzuki H., Kaifu, N., Ishikawa, S., \& Ohishi, M. 1990, ApJ, 361, 318

This preprint was prepared with the AAS IATEX macros v5.2.

Table 1. Source coordinate

\begin{tabular}{lll}
\hline \hline \multicolumn{1}{c}{ Source } & $\alpha(\mathrm{J} 2000)$ & \multicolumn{1}{c}{$\delta(\mathrm{J} 2000)$} \\
Name & $(\mathrm{h} \mathrm{m} \mathrm{s})$ & $\left({ }^{\circ},{ }^{\prime \prime}\right)$ \\
\hline CB130-3 & $18: 16: 17.9$ & $-02: 16: 41$ \\
L673-SMM4 & $19: 20: 24.6$ & $+11: 24: 34$ \\
\hline
\end{tabular}


Table 2. List of molecular lines observed with the NRO $45 \mathrm{~m}$ telescope

\begin{tabular}{|c|c|c|c|c|c|c|c|c|c|}
\hline Source Name & Line & $\nu(\mathrm{MHz})$ & $S_{u l}^{\mathrm{a}}$ & $\mu(\mathrm{D})^{\mathrm{b}}$ & Reference & $T_{B}(\mathrm{~K})$ & $v_{l s r}\left(\mathrm{~km} \mathrm{~s}^{-1}\right)$ & $\Delta v\left(\mathrm{~km} \mathrm{~s}^{-1}\right)$ & $T_{r m s}(\mathrm{~K})$ \\
\hline \multirow[t]{7}{*}{ CB130-3 } & $\operatorname{CCS}\left(J_{N}=43-3_{2}\right)$ & 45379.033 & 3.97 & 2.81 & 1,2 & $2.26(33)$ & $7.22(3)$ & $0.41(7)$ & 0.16 \\
\hline & $\mathrm{HC}_{3} \mathrm{~N}(J=5-4)$ & 45490.316 & 5.00 & 3.72 & 3 & $2.83(24)$ & $7.22(4)$ & $0.81(8)$ & 0.13 \\
\hline & $\mathrm{HC}_{5} \mathrm{~N}(J=17-16)$ & 45264.721 & 17.00 & 4.33 & 4 & $0.83(47)$ & $7.40(8)$ & $0.24(17)$ & 0.13 \\
\hline & $\mathrm{H}^{13} \mathrm{CO}^{+}(J=1-0)$ & 86754.330 & 1.00 & 4.07 & 5 & $1.35(16)$ & $7.31(3)$ & $0.44(6)$ & 0.08 \\
\hline & $\operatorname{DNC}(J=1-0)$ & 76305.717 & 1.00 & 3.05 & 6 & $0.48(12)$ & $7.17(12)$ & $0.94(27)$ & 0.09 \\
\hline & $\mathrm{HN}^{13} \mathrm{C}(J=1-0)$ & 87090.859 & 1.00 & 3.05 & 6 & $0.41(11)$ & $7.11(10)$ & $0.72(22)$ & 0.08 \\
\hline & $\mathrm{N}_{2} \mathrm{H}^{+}(J=1-0)^{\mathrm{c}}$ & 93173.777 & 1.00 & 3.40 & 7 & 0.91 & $7.24(4)$ & $0.36(5)$ & 0.11 \\
\hline \multirow[t]{7}{*}{ L673-SMM4 } & $\operatorname{CCS}\left(J_{N}=4_{3}-3_{2}\right)$ & 45379.033 & 3.97 & 2.81 & 1,2 & $2.19(26)$ & $6.59(5)$ & $0.76(11)$ & 0.19 \\
\hline & $\mathrm{HC}_{3} \mathrm{~N}(J=5-4)$ & 45490.316 & 5.00 & 3.72 & 3 & $4.24(27)$ & $6.65(3)$ & $1.02(8)$ & 0.14 \\
\hline & $\mathrm{HC}_{5} \mathrm{~N}(J=17-16)$ & 45264.721 & 17.00 & 4.33 & 4 & $1.51(31)$ & $6.59(7)$ & $0.62(15)$ & 0.17 \\
\hline & $\mathrm{H}^{13} \mathrm{CO}^{+}(J=1-0)$ & 86754.330 & 1.00 & 4.07 & 5 & $1.15(10)$ & $6.68(4)$ & $0.90(9)$ & 0.09 \\
\hline & $\operatorname{DNC}(J=1-0)$ & 76305.717 & 1.00 & 3.05 & 6 & $1.36(13)$ & $6.57(5)$ & $1.02(11)$ & 0.11 \\
\hline & $\mathrm{HN}^{13} \mathrm{C}(J=1-0)$ & 87090.859 & 1.00 & 3.05 & 6 & $0.77(11)$ & $6.60(6)$ & $0.86(14)$ & 0.09 \\
\hline & $\mathrm{N}_{2} \mathrm{H}^{+}(J=1-0)^{\mathrm{c}}$ & 93173.777 & 1.00 & 3.40 & 7 & 1.65 & $6.65(5)$ & $0.72(7)$ & 0.17 \\
\hline
\end{tabular}

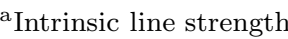

b Dipole moment

${ }^{\mathrm{c}}$ Brightness temperature of the main hyperfine component. Line widths and peak velocities are derived from the hyperfine fitting method (see text).

Note. - Observed positions are $\left(-40^{\prime \prime},-40^{\prime \prime}\right)$ and $\left(40^{\prime \prime}, 40^{\prime \prime}\right)$ for CB130-3 and L673-SMM4, respectively.

The numbers in parenthesis represent three times the standard deviation in the Gaussian fit in units of the last significant digits.

References. - 1: Murakami (1990); 2: Yamamoto et al. (1990); 3: Lafferty \& Lovas (1978); 4: Alexander et al. (1976); 5: Haese \& Woods (1979)); 6: Blackman et al. (1976); 7: Havenith et al. (1990) 
Table 3. Parameters for the $\mathrm{NH}_{3}$ lines observed with the MPIfR $100 \mathrm{~m}$ telescope

\begin{tabular}{|c|c|c|c|c|c|c|c|}
\hline $\begin{array}{l}\text { Source } \\
\text { Name }\end{array}$ & $\begin{array}{l}T_{B}{ }^{\mathrm{a}} \\
(\mathrm{K})\end{array}$ & $\begin{array}{c}v_{l s r} \\
\left(\mathrm{~km} \mathrm{~s}^{-1}\right)\end{array}$ & $\begin{array}{c}\Delta v \\
\left(\mathrm{~km} \mathrm{~s}^{-1}\right)\end{array}$ & $\tau_{\text {main }} \mathrm{b}$ & $\begin{array}{c}N\left[\mathrm{NH}_{3}\right] \\
\left(10^{14} \mathrm{~cm}^{-3}\right)\end{array}$ & $\begin{array}{l}T_{e x} \\
(\mathrm{~K})\end{array}$ & $\begin{array}{r}T_{r m s} \\
(\mathrm{~K})\end{array}$ \\
\hline CB130-3c & 1.07 & $7.26(3)$ & $0.78(6)$ & 0.3 & $1.63\left({ }_{-26}^{+125}\right)$ & 6.5 & 0.14 \\
\hline L673-SMM4 & 2.8 & $6.72(1)$ & $0.43(3)$ & $1.8(4)$ & $5.1(14)$ & $6.6(15)$ & 0.11 \\
\hline $\mathrm{L} 492^{\mathrm{d}}$ & 2.5 & $7.81(1)$ & $0.30(2)$ & $3.5(5)$ & $5.5(12)$ & $5.6(10)$ & 0.13 \\
\hline TMC- $1^{\mathrm{d}}$ & 1.91 & $5.99(2)$ & $0.50(4)$ & $1.1(5)$ & $3.2(17)$ & $6.2(28)$ & 0.16 \\
\hline
\end{tabular}

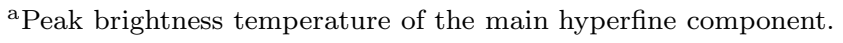

${ }^{b}$ Optical depth of the main hyperfine components.

${ }^{\mathrm{c}}$ Hyperfine fitting was not successful. Only the main hyperfine component was fitted to the Gaussian profile, and the column density was derived by assuming the excitation temperature of $6.5 \mathrm{~K}$ (see text).

${ }^{\mathrm{d}}$ See Appendix.

Note. - The numbers in parenthesis represent three times the standard deviation in the Gaussian fit in units of the last significant digits.

Table 4. Column densities toward CB130-3 and L673-SMM4

\begin{tabular}{lll}
\hline \hline Molecule & \multicolumn{1}{c}{ CB130-3 } & L673-SMM4 \\
\hline $\mathrm{CCS}$ & $2.2_{-0.2}^{+0.6}$ & $3.8_{-0.4}^{+0.9}$ \\
$\mathrm{HC}_{3} \mathrm{~N}$ & $2.8_{-0.4}^{+1.0}$ & $6.8_{-1.5}^{+5.8}$ \\
$\mathrm{HC}_{5} \mathrm{~N}$ & $0.81_{-0.15}^{+0.22}$ & $4.3_{-0.8}^{+1.3}$ \\
$\mathrm{H}^{13} \mathrm{CO}^{+}$ & $0.072_{-0.018}^{+0.034}$ & $0.123_{-0.030}^{+0.055}$ \\
$\mathrm{DNC}^{+0.016}$ & $0.20_{-0.12}^{+0.16}$ & $0.74_{-0.46}^{+0.65}$ \\
$\mathrm{HN}^{13} \mathrm{C}$ & $0.156_{-0.10}^{+0.13}$ & $0.38_{-0.25}^{+0.34}$ \\
$\mathrm{~N}_{2} \mathrm{H}^{+}$ & $0.22_{-0.03}^{+0.04}$ & $1.21_{-0.19}^{+0.20}$ \\
$\mathrm{NH}_{3}$ & $16.3_{-2.6}^{+12.5}$ & $51_{-14}^{+14}$ \\
\hline
\end{tabular}

Note. - The column densities are in unit of $10^{13} \mathrm{~cm}^{-2}$.

Table 5. Molecular abundance ratios

\begin{tabular}{lccccccccc}
\hline \hline Molecule & L1521E & L1521B & L1495B & TMC-1(CP) & CB130-3 & L492 & L673-SMM4 & L1544 & L1498 \\
\hline $\mathrm{DNC}^{2} \mathrm{HN}^{13} \mathrm{C}$ & 0.66 & 0.70 & $<0.66$ & 1.25 & $1.28_{-0.05}^{+0.27}$ & 1.27 & $1.96_{-0.01}^{+0.32}$ & 3.0 & 1.91 \\
$\mathrm{NH}_{3} / \mathrm{CCS}$ & 2.6 & 3.5 & 3.8 & 4.8 & $7.6_{-0.4}^{+3.0}$ & 10.4 & $13.6_{-2.5}^{+0.3}$ & 15 & 25 \\
\hline
\end{tabular}

Note. - For CB130-3 and L673-SMM4, the results of the present study are given. For the other sources, the values are taken from Hirota \& Yamamoto (2006) and references therein, except for the $\mathrm{NH}_{3} / \mathrm{CCS}$ ratios in TMC-1(CP) and L492 (see Appendix). 


\section{A. Follow-up Observations of the $\mathrm{NH}_{3}$ Lines toward L492 and TMC-1}

In Figure 4, we plot the relationship between the $\mathrm{DNC} / \mathrm{HN}^{13} \mathrm{C}$ and $\mathrm{NH}_{3} / \mathrm{CCS}$ ratios. The original plot was reported by Hirota et al. (2001) and we have here revised it by employing the present results for CB130-3 and L673-SMM4. In addition, we have also involved the result of the L492 observation in the present study. For L492, the observed position of the $\mathrm{NH}_{3}$ line was shifted from the CCS peak position (Hirota \& Yamamoto 2006). Therefore, we have employed the $\mathrm{NH}_{3}$ data toward the CCS peak position $\left(40^{\prime \prime}, 0^{\prime \prime}\right)$ of L492 observed with the MPIfR $100 \mathrm{~m}$ telescope as described in section 2. In addition, we have also replaced the $\mathrm{NH}_{3}$ data for the cyanopolyyne peak of TMC-1 with that obtained with the MPIfR $100 \mathrm{~m}$ telescope. The result of the $\mathrm{NH}_{3}$ observation is summarized in Table 3 and Figure 3 , The $\mathrm{NH}_{3}$ column densities in L492 and TMC-1 become larger by a factor of 1.6 and 1.7, respectively. For the $\mathrm{DNC} / \mathrm{HN}^{13} \mathrm{C}$ ratios, we have employed recently observed data reported by Hirota et al. (2002), Hirota et al. (2003), Hirota et al. (2004), Hirota \& Yamamoto (2006), and the present study. 


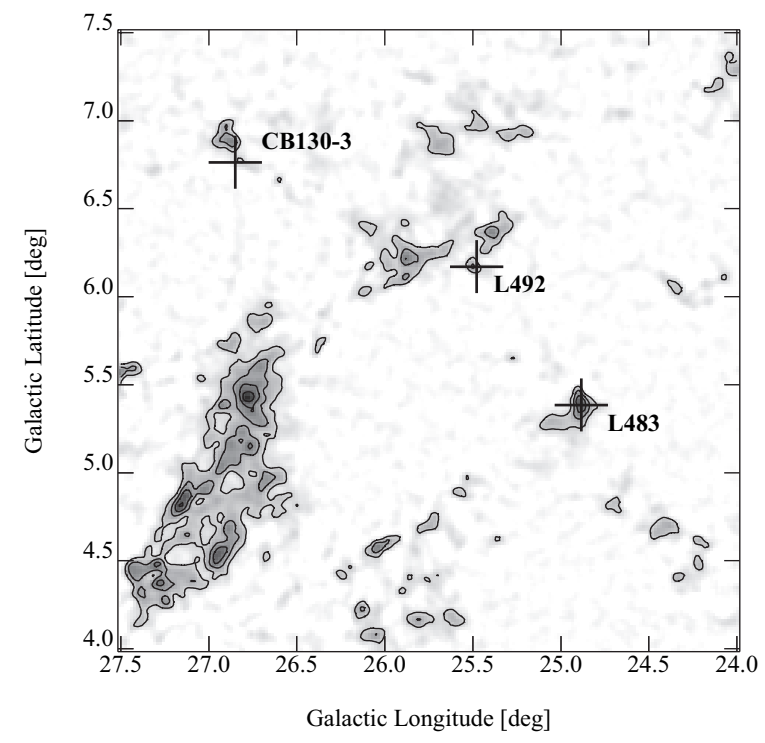

Fig. 1.- Distribution of dark clouds in the GF4 region (Schneider \& Elmegreen 1979). Three dense cores, CB130-3, L492, and L483, are aligned from the top-left (north) to bottomright (south) direction. The map shows the visual extinction $A_{V}$ composed from the 2MASS extinction data (Dobashi 2011). The contour levels are $A_{V}=2,4,6,8$, and 10 mag. 

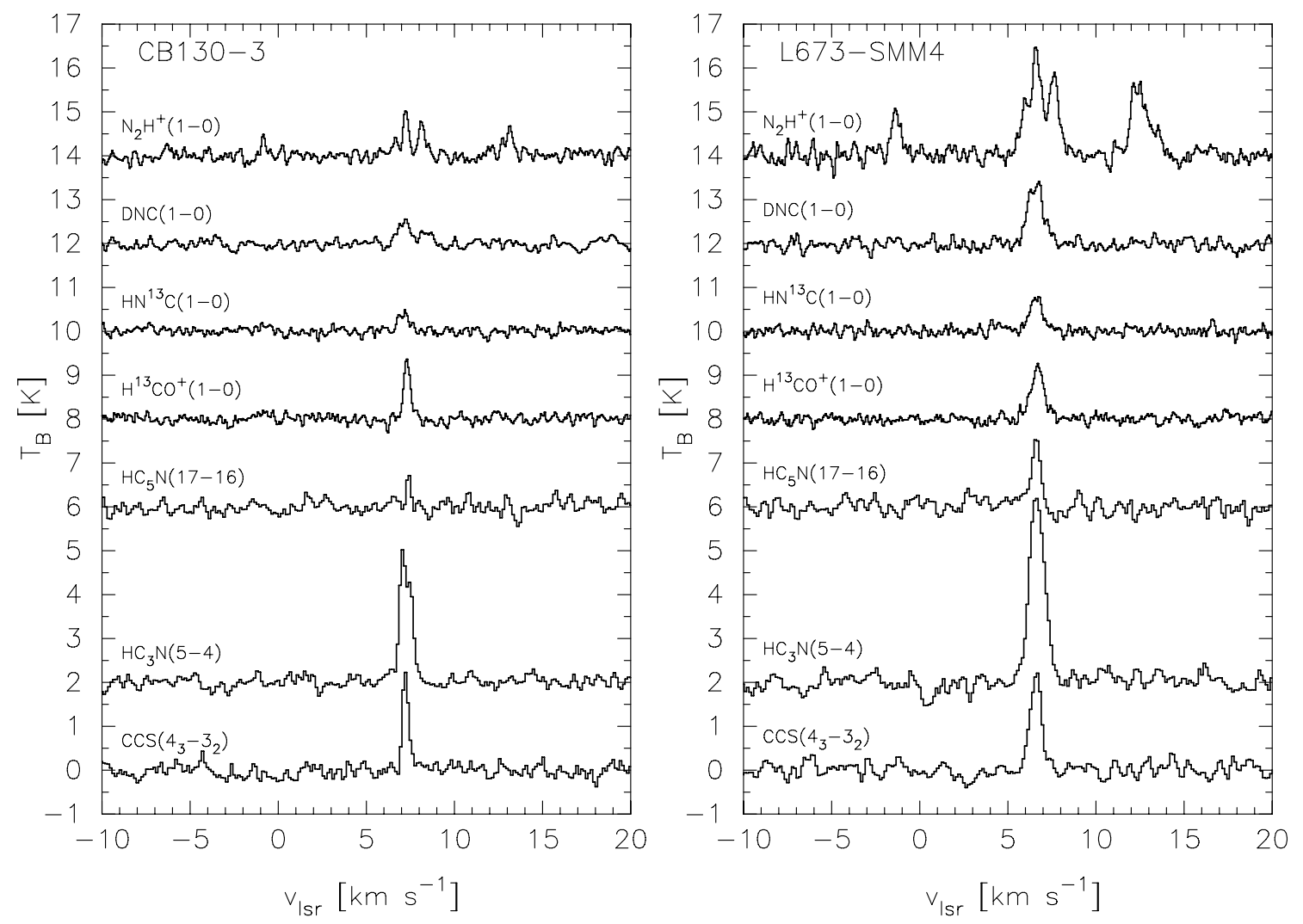

Fig. 2.- Line profiles observed toward CB130-3 and L673-SMM4. 

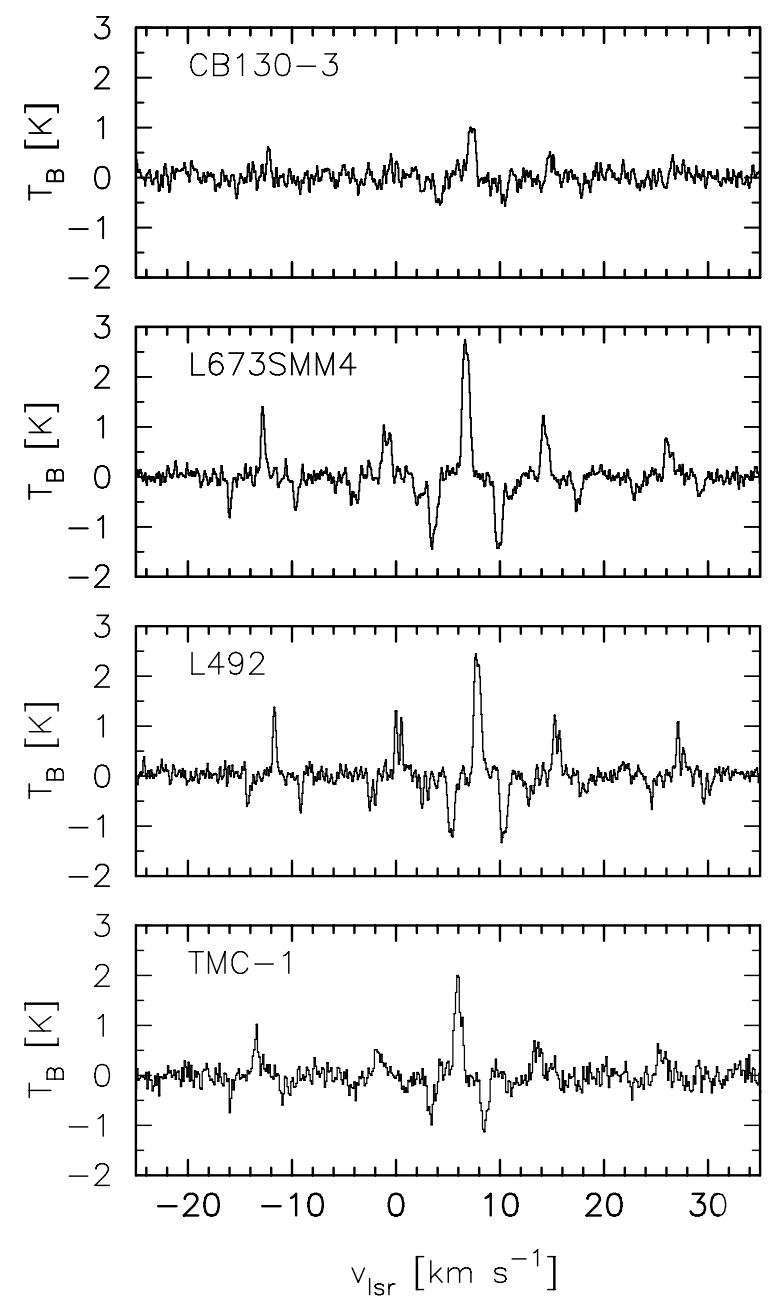

Fig. 3.- Spectra of the $\mathrm{NH}_{3}(1,1)$ lines. The absorption-like features below the baseline are due to artifacts of the frequency switching observations. The results for L492 and TMC-1 are also plotted (see Appendix). 


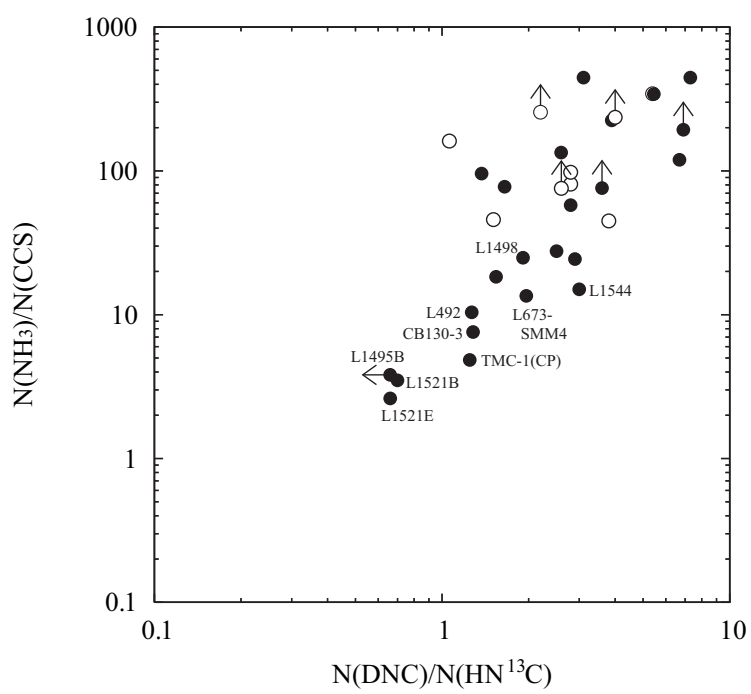

Fig. 4.- Relationship between the abundance ratios of $\mathrm{DNC} / \mathrm{HN}^{13} \mathrm{C}$ and $\mathrm{NH}_{3} / \mathrm{CCS}$. Filled and open symbols represent the abundance ratios for starless and star-forming cores, respectively. The data were originally presented in Hirota et al. (2001). We have revised the data for CCPRs (Hirota et al. 2002, 2003, 2004; Hirota \& Yamamoto 2006).

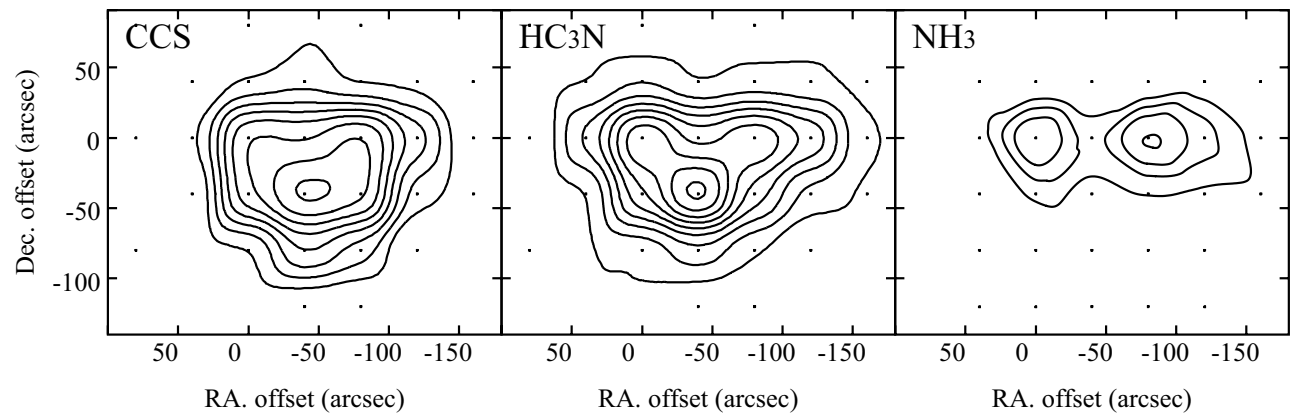

Fig. 5.- Integrated intensity maps of CB130-3. The reference position is listed in Table 1. The velocity range of integration, lowest contour levels, and contour intervals are (6.4$7.8 \mathrm{~km} \mathrm{~s}^{-1}, 0.23 \mathrm{~K} \mathrm{~km} \mathrm{~s}^{-1}, 0.11 \mathrm{~K} \mathrm{~km} \mathrm{~s}^{-1}$ ) for CCS, (6.4-8.0 $\mathrm{km} \mathrm{s}^{-1}, 0.23 \mathrm{~K} \mathrm{~km} \mathrm{~s}^{-1}$, $\left.0.23 \mathrm{~K} \mathrm{~km} \mathrm{~s}^{-1}\right)$ for $\mathrm{HC}_{3} \mathrm{~N}$, and $\left(6.5-8.5 \mathrm{~km} \mathrm{~s}^{-1}, 0.60 \mathrm{~K} \mathrm{~km} \mathrm{~s}^{-1}, 0.30 \mathrm{~K} \mathrm{~km} \mathrm{~s}^{-1}\right)$ for the main hyperfine component of $\mathrm{NH}_{3}(1,1)$. 


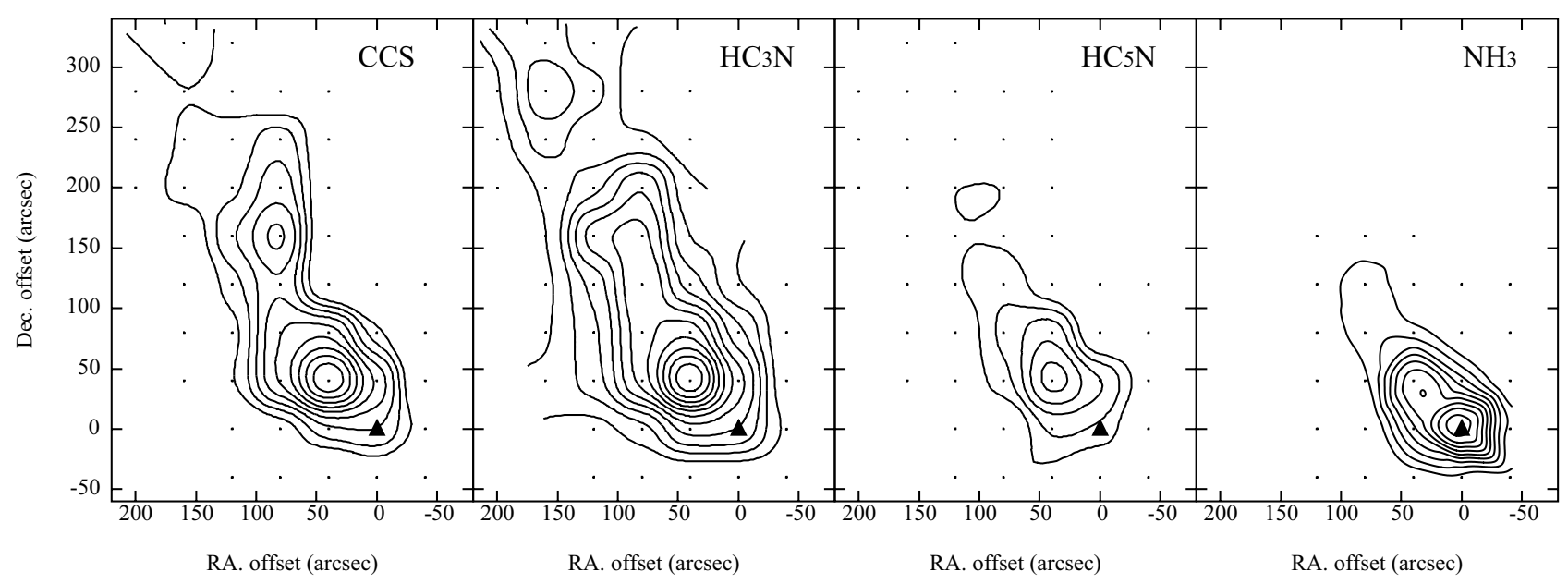

Fig. 6. - Integrated intensity maps of L673-SMM4. The reference position is listed in Table 1. The velocity range of integration, lowest contour levels, and contour intervals are (5.8-7.4 $\left.\mathrm{km} \mathrm{s}^{-1}, 0.29 \mathrm{~K} \mathrm{~km} \mathrm{~s}^{-1}, 0.14 \mathrm{~K} \mathrm{~km} \mathrm{~s}^{-1}\right)$ for CCS, $\left(5.6-7.8 \mathrm{~km} \mathrm{~s}^{-1}, 0.34 \mathrm{~K} \mathrm{~km} \mathrm{~s}^{-1}\right.$, $\left.0.34 \mathrm{~K} \mathrm{~km} \mathrm{~s}^{-1}\right)$ for $\mathrm{HC}_{3} \mathrm{~N}$, (5.8-7.4 $\left.\mathrm{km} \mathrm{s}^{-1}, 0.29 \mathrm{~K} \mathrm{~km} \mathrm{~s}^{-1}, 0.14 \mathrm{~K} \mathrm{~km} \mathrm{~s}^{-1}\right)$ for $\mathrm{HC}_{5} \mathrm{~N}$, and $\left(6.0-7.5 \mathrm{~km} \mathrm{~s}^{-1}, 0.60 \mathrm{~K} \mathrm{~km} \mathrm{~s}^{-1}, 0.30 \mathrm{~K} \mathrm{~km} \mathrm{~s}^{-1}\right)$ for the main hyperfine component of $\mathrm{NH}_{3}(1,1)$. A triangle in each map represents the submillimeter continuum source SMM4 (Visser et al. 2002). 


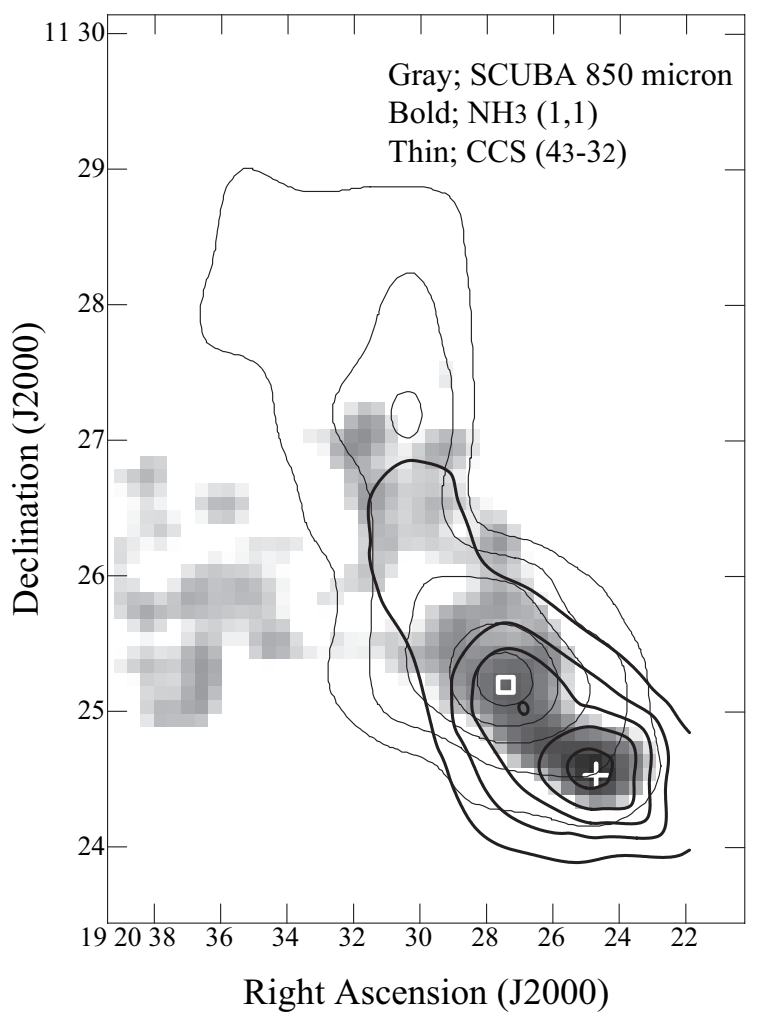

Fig. 7.- Integrated intensity maps of L673-SMM4 superposed on the dust continuum map taken from the SCUBA Legacy Catalog (Di Francesco et al. 2008). The gray scale represents the dust continuum emission at $850 \mu \mathrm{m}$. The bold and thin contours show the integrated intensity of the CCS and $\mathrm{NH}_{3}$ lines, respectively. The lowest contour levels and contour intervals are $\left(0.29 \mathrm{~K} \mathrm{~km} \mathrm{~s}^{-1}, 0.29 \mathrm{~K} \mathrm{~km} \mathrm{~s}^{-1}\right)$ for CCS and $\left(0.60 \mathrm{~K} \mathrm{~km} \mathrm{~s}^{-1}, 0.60 \mathrm{~K} \mathrm{~km} \mathrm{~s}^{-1}\right)$ for the main hyperfine component of $\mathrm{NH}_{3}(1,1)$. A cross and a square indicate the positions of the $\mathrm{NH}_{3}$ and CCS peaks, respectively. 